\title{
Insomnia May Mediate the Relationship Between Stress and Anxiety: A Cross-Sectional Study in University Students
}

This article was published in the following Dove Press journal: Nature and Science of Sleep

\author{
Md Dilshad Manzar (iD) \\ Mohammed Salahuddin (iD) 2,3 \\ Seithikurippu R Pandi-Perumal (iD) ${ }^{4}$ \\ Ahmed S Bahammam iD ${ }^{5,6}$ \\ 'Department of Nursing, College of \\ Applied Medical Sciences, Majmaah \\ University, Al Majmaah II952, Saudi \\ Arabia; ${ }^{2}$ Department of Pharmacy, \\ College of Medicine and Health Sciences, \\ Mizan-Tepi University (Mizan Campus), \\ Mizan-Aman, Ethiopia; ${ }^{3}$ Pharmacology \\ Division, Department of BioMolecular \\ Sciences, University of Mississippi, \\ Oxford, MS, USA; ${ }^{4}$ Somnogen Canada \\ Inc, Toronto, ON, Canada; ${ }^{5}$ The \\ University Sleep Disorders Center, \\ College of Medicine, King Saud \\ University, Riyadh II324, Saudi Arabia; \\ ${ }^{6}$ National Plan for Science and \\ Technology, College of Medicine, King \\ Saud University, Riyadh, Saudi Arabia
}

Correspondence: Md Dilshad Manzar Department of Nursing, College of Applied Medical Sciences, Majmaah University, Al Majmaah I 1952, Saudi Arabia Tel +966-551923468

Email m.manzar@mu.edu.sa
Background: High perceived stress and anxiety disorders are usually comorbid with each other, with stress often sequentially preceding the development of anxiety. While prior findings showed a causal role of sleep problems in anxiety, no study has assessed the role of insomnia as a mediator in the relationship between stress and anxiety.

Methods: A cross-sectional study on university students $(n=475$, age $=21.1+2.6$ years $)$ was conducted over 3 months. Participants completed self-report measures of Leeds Sleep Evaluation Questionnaire-Mizan (LSEQ-M), Perceived Stress Scale-10 (PSS-10), generalized anxiety disorder-7 scale (GAD-7), and a sociodemographic tool. The mediation effect model given by Baron and Kelly was used to determine the relationship.

Results: The prevalence of insomnia and anxiety disorder was $43.6 \%$ and $21.9 \%$, respectively. Stress was significantly associated with LSEQ-M (insomnia measure) $(b=-.44, \mathrm{SE}=$ $0.16, \mathrm{p}<0.01)$, and high levels of anxiety $(\mathrm{b}=0.25, \mathrm{SE}=0.03, \mathrm{p}<0.01)$. The indirect effect of stress on anxiety through LSEQ-M (insomnia measure) was significant ( $95 \%$ confidence interval $[0.01,0.04])$. However, the indirect effect of anxiety on stress through LSEQ-M (insomnia measure) was non-significant (95\% confidence interval [-.01, 0.04]).

Conclusions: Students having higher perceived stress levels and comorbid insomnia were also likely to have a higher anxiety level.

Keywords: stress, anxiety, insomnia, mediator

\section{Introduction}

One-third of the population is reported to have anxiety disorders at some point in time during their lifespan. ${ }^{1}$ Anxiety disorders have a high economic burden because they are associated with high utilization of healthcare services use and a substantial level of impairments. The situation is challenging because of the under-reporting of treatment, improper diagnoses, and non-use/availability of the proper treatment. ${ }^{1}$ Stress and anxiety are major psychiatric health issues, both of which are highly prevalent in the university students' populations and most often coexist. ${ }^{2}$ Studies using animal models of stress have shown that stress is involved in increasing or initiating anxiety. ${ }^{3,4}$ Social deprivation stress causes surfacing of anxiety, while chronic psychosocial stress increases anxiety-like behavior in mice.,

A higher level of stress is associated with poor sleep quality in college/university students in different countries. ${ }^{5-7}$ Stress is associated with shorter sleep duration and longer sleep latency in adolescents. ${ }^{8}$ Some people have a trait-like reactivity to stress that may express in sleep problems (like insomnia-type symptoms) in response to 
stress. ${ }^{9}$ Such a sleep-reactivity is heritable susceptibility to sleep problems including insomnia. ${ }^{10,11}$ Such people are more prone to sleep disturbances when exposed to precipitating factors like caffeine consumption, circadian disruptions, etc. ${ }^{9}$ Stress-related sleep reactivity and anxiety symptoms may work as distinguishing features between insomniacs and good sleepers. ${ }^{12}$ Estimates show that $\sim 24-36 \%$ of the population with anxiety disorders were reported to have comorbid insomnia. ${ }^{13}$ In university students, clinical anxiety is more prevalent in those with insomnia, poor sleep quality, and excessive daytime sleepiness. ${ }^{14}$ All this evidence indicates that these three pairs, 1) stress and anxiety, 2) stress and insomnia, and 3) anxiety and insomnia, are often comorbid. However, a major shortcoming of existing studies is that they have not assessed insomnia, stress, and anxiety in one model, limiting the generalizability of the relationship between these three conditions. Thus, studies that account for the mediator and moderator relationship in a model are needed to understand these processes. Stress and anxiety disorders typically develop by late childhood and early adolescence, whereas sleep problems like insomnia increase during the transition from adolescence to adulthood, mostly driven by environmental and physiological changes. ${ }^{15-18}$ Thus, an examination of associations between insomnia, stress, and anxiety may be particularly useful in understanding their meditational role. Therefore, the research hypothesis was that stress and anxiety are associated and that the association is mediated by insomnia.

\section{Materials and Methods}

\section{Participants and Procedure}

A sample size of 507 students was determined based on $5 \%$ margin of error, $99 \%$ confidence level, population size (5553 students at Mizan campus of the Mizan-Tepi University (MTU) at the time of study in 2018), and $30 \%$ of the expected response distribution for anxiety. ${ }^{19}$ From a list of 650 enrolled students; 47 chose not to participate when approached, 78 did not provide responses or did not report for the interview, 28 were excluded after screening, and 22 responses were deleted from analysis because of missing responses for all (or most) of the items of all of the three questionnaires, ie, LSEQ-M, Gad-7, and PSS-10. Participants recorded their responses in hard copies of the survey questionnaire package during individual meetings with the researchers. Finally, 475 collegiate young adults (age: $21.1 \pm 2.6$ years) from MTU, MizanAman, Ethiopia, were included in this cross-sectional study. Students were enrolled in different courses at the university. The exclusion was based on the personal account of memory problems and those taking medicines for neuropsychiatric conditions. This was done to minimize the chances of memory-related bias affecting the quality of the study.

The study was reviewed and approved by the institutional ethical committee at the College of Health Sciences, Mizan-Tepi University, Ethiopia. All participants were given a brief explanation about the procedures to be followed before the commencement of the data collection. Participants were also informed that 1) participation was voluntary with the right to discontinue at any stage, 2) no direct benefits and $\operatorname{risk}(\mathrm{s})$ were associated, 3) standard procedures were in place to keep personal information confidential, 4) no fee to be paid, and 5) study results will be summarized using group properties using tables and figures without revealing individual's details. In compliance with the Helsinki declaration, informed written consent was given by the participants. Generalized anxiety disorder-7 scale (GAD-7), Leeds Sleep Evaluation Questionnaire-Mizan (LSEQ-M), and Perceived Stress Scale-10 (PSS-10) were used. As all participants were students of an Ethiopian government university with English as the medium of instruction; therefore, all questionnaire tools were used in English.

\section{Measures}

\section{Generalized Anxiety Disorder-7 Scale (GAD-7)}

GAD-7 measures respondents' anxiety-related symptoms over the last 2 weeks. It is a brief measure with seven items; each of these items is scored on an ordinal scale of $0-3$, where ' 0 ' represents "not at all" and ' 3 ' represents "nearly every day". 20,21 A total score for the scale henceforth is referred to as GAD total score; it is generated by adding scores for all the seven items. GAD total score may range from 0 to 21 ; a higher score implies increasing severity of anxiety complaints. ${ }^{20}$ GAD-7 has been found to have adequate diagnostic validity for screening anxiety disorder at the cut-off score of 10 with sufficient accuracy. $^{22}$

\section{Perceived Stress Scale- 10 (PSS- I0)}

PSS-10 measures respondents' stress-related symptoms during the last month immediately preceding the screening. PSS10 is a brief measure to assess the level of psychological stress with 10-items; each of these 10-items is scored on an ordinal scale of $0-3$, where ' 0 ' represent "never" and ' 3 ' represents 
"very often". ${ }^{23}$ A total score for the scale henceforth is referred to as the PSS-10 score; it is generated by adding scores for all the 10-items; scores for four of items are reversed before addition. The PSS-10 total score may range from 0 to 40 ; a higher score implies increasing severity of stress complaints. $^{23}$ The PSS-10 has been found to have adequate psychometric validation in the Ethiopian collegiate young adults. $^{24}$

\section{Leeds Sleep Evaluation Questionnaire-Mizan (LSEQ-M)}

LSEQ-M in an adapted English version of the original LSEQ, which has been found to have adequate diagnostic validity for screening insomnia in collegiate young adults $^{25-27}$ LSEQ-M has 10-self reported items that are scored on a $100 \mathrm{~mm}$ visual analog scale (VAS). ${ }^{26}$ A total score for the scale henceforth is referred to as the LSEQ-M total score; it is generated by adding scores for all the 10items. $^{26}$ All the individual item scores are divided by 10 before addition to get the LSEQ-M total score. ${ }^{26}$ Unlike, GAD-7 and PSS-10, lower scores for LSEQ-M are indicative of increasing severity of its measure, ie, insomnia. ${ }^{26}$

\section{Statistical Analysis}

SPSS version 23.0, along with macros SPSS PROCESS, was used to perform the statistical analysis. Descriptive statistics, ie, mean \pm Standard deviation (SD), frequency, and percentage were used for presenting participants' characteristics. The mediation effect model given by Baron and Kelly was implemented to determine the relationship. ${ }^{28}$ The mediating effect of a single mediator LSEQ-M (insomnia) on the relationship of psychological stress and anxiety was evaluated using SPSS PROCESS. ${ }^{29,30}$ Total scores of LSEQ-M, PSS-10, and GAD-7 were used as measures of insomnia, stress, and anxiety levels, respectively, in this study. Total scores of all these three measures were used as continuous measures. The Sobel test with criteria of absence of 0 in the bootstrapped confidence interval (CI) was used to indicate the significance of the indirect effect.

\section{Results}

\section{Participants' Characteristics}

The average age of the participating collegiate young adults in this study was $21.1 \pm 2.6$ years (Table 1). Most of the participants were males $(80.0 \%)$ (Table 1$)$. The mean GAD7 total score, PSS-10 total score, and LSEQ-M total score were $7.3 \pm 4.2,20.1 \pm 5.5$, and $57.8 \pm 20.0$, respectively
(Table 1). The prevalence of insomnia and anxiety disorders was $43.6 \%$ and $21.9 \%$, respectively (Table 1 ).

\section{Insomnia as a Mediator in the Effect of Stress on Anxiety}

The results of the mediation analysis to assess the effect of stress on anxiety through LSEQ-M (insomnia measure) are shown in Figure 1. The overall model explained $15.94 \%$ of the variance in stress $\left(\mathrm{R}^{2}=0.1594, \mathrm{~F}[1,472]=44.76, \mathrm{p}<0.001\right)$. Stress was a negative and significant predictor of LSEQ-M (b $=-.44$, standard error $(\mathrm{SE})=0.16, \mathrm{p}<0.01)$, indicating that those with higher stress levels were likely to have a higher incidence of insomnia. LSEQ-M was also a negative predictor of anxiety $(b=-.04, S E=0.01, p<0.001)$, indicating that those with insomnia were likely to have anxiety. The direct effect of stress on anxiety remained positive and significant after controlling for the effect of LSEQ-M $(b=0.25, \mathrm{SE}=0.03, \mathrm{p}<$ 0.001). The indirect effect was statistically significant: $95 \% \mathrm{CI}$ $=(0.01-0.04)$, implying that insomnia was a significant mediator in the effect of stress on anxiety.

\section{Insomnia Was Not a Mediator in the Effect of Anxiety on Stress}

The results of the mediation analysis to assess the effect of anxiety on stress through LSEQ-M (insomnia measure) are shown in Figure 2. Insomnia did not mediate the reverse relation, ie, anxiety on stress: LSEQ-M was not a significant predictor of stress $(b=-0.01, \mathrm{SE}=0.01$,

Table I Participants' Characteristics

\begin{tabular}{|l|l|}
\hline Characteristics & Mean \pm SD/Frequency (Percentage) \\
\hline Age (yr) & $21.1 \pm 2.6$ \\
\hline $\begin{array}{l}\text { Gender } \\
\text { Female } \\
\text { Male }\end{array}$ & $\begin{array}{l}95(20.0) \\
380(80.0)\end{array}$ \\
\hline $\begin{array}{l}\text { GAD-7 total score } \\
\text { PSS-I0 total score } \\
\text { LSEQ-M total score }\end{array}$ & $\begin{array}{l}7.3 \pm 4.2 \\
20.1 \pm 5.5\end{array}$ \\
\hline $\begin{array}{l}\text { Insomnia } \\
\text { Yes }\end{array}$ & $207(43.6)$ \\
No & $268(56.4)$ \\
\hline Anxiety disorder & \\
Yes & $104(21.9)$ \\
No & $371(78.1)$ \\
\hline
\end{tabular}

Abbreviations: SD, standard deviation; GAD-7, generalized anxiety disorder-7 scale; PSS-10, Perceived Stress Scale-10; LSEQ-M, Leeds Sleep Evaluation Questionnaire-Mizan. 


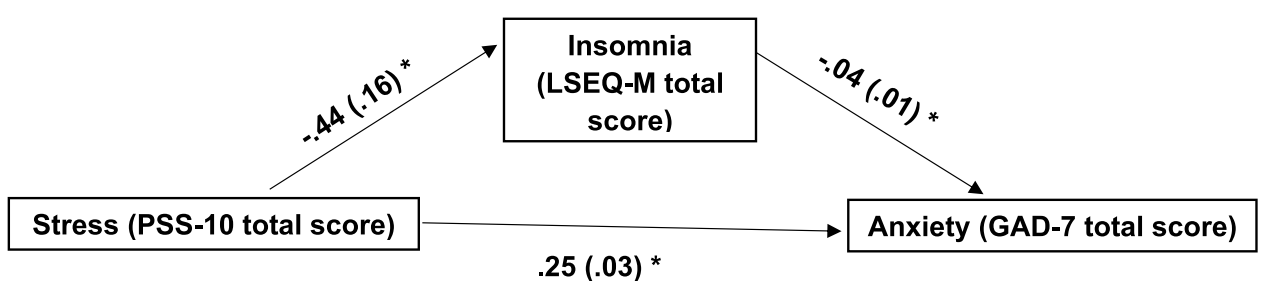

Figure I Model with LSEQ-M (insomnia measure) as a mediator in the effect of stress on anxiety.

Notes: First values are the unstandardized coefficients and second values within brackets are the standard errors; $* p<0.01$. The indirect effect of stress on anxiety through LSEQ-M (insomnia measure) was significant ( $95 \%$ confidence interval [0.01, 0.04]).

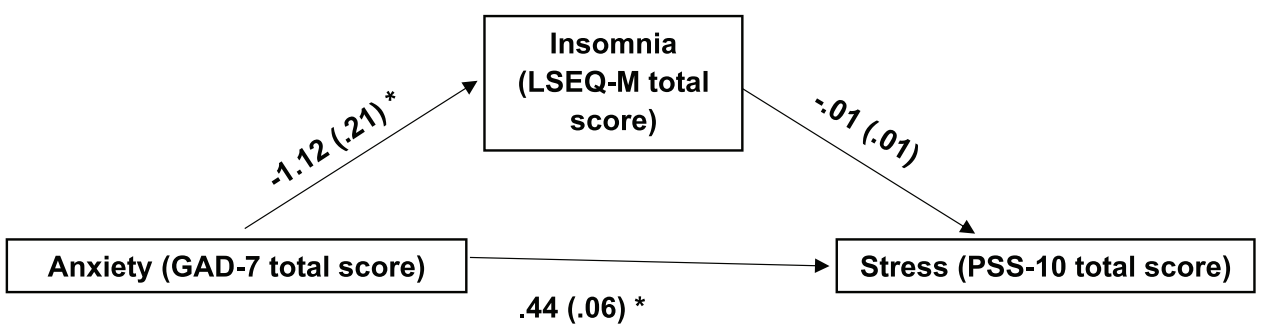

Figure 2 Model with LSEQ-M (insomnia measure) as a mediator in the effect of anxiety on stress.

Notes: First values are the unstandardized coefficients and second value within brackets are the standard errors; $* p<0.01$. The indirect effect of anxiety on stress through LSEQ-M (insomnia measure) was non-significant ( $95 \%$ confidence interval $[-0.01,0.04]$ ). And, LSEQ-M (insomnia measure) was not a significant predictor of stress $(b=-.0 \mathrm{I}, \mathrm{SE}=0.0 \mathrm{I}, \mathrm{p}=0.35)$.

$\mathrm{p}=35)$, and the indirect effect of anxiety on stress through LSEQ-M was non-significant (95\% confidence interval $[-0.01,0.04])$.

\section{Discussion}

The current study examined insomnia as a mediator between stress and anxiety in university students. The bidirectional relationship between chronic stress and anxiety has already been established, ${ }^{30,31}$ with most of the previous studies emphasizing the underlying neurobiological mechanisms. ${ }^{32}$ However, there is a lack of studies on the underlying psychological mechanisms. To this end, we sought to examine the role of insomnia in mediating the relationship between stress and anxiety. Consistent with our hypothesis, the results provided evidence in support of insomnia as a mediator in the relationship between stress and anxiety symptoms. This implies that students having a higher perceived stress level, and insomnia were also likely to have anxiety. To the best of our knowledge, this is the first study to examine the mediator role of insomnia in the comorbidity of stress and anxiety.

To establish the mediating effect of a factor, it is desirable to use a longitudinal study design, this may provide more robust evidence. However, it is an accepted practice to employ Baron and Kelly's approach to build initial evidence about the mediating effect of a factor using a cross-sectional design. ${ }^{28}$ Moreover, longitudinal designs may raise ethical concerns as patient groups, ie, in this case, students with stress and/or anxiety and/or insomnia may have to be followed without allowing them to seek access to treatment for long periods. In such situations, Baron and Kelly's approach provides a more ethically viable approach to building initial evidence about mediating factors without the need to keep participants away from access to treatment. ${ }^{28}$ Therefore, in this study, we used this method. The current study revealed that insomnia was not a significant mediator in the relationship of anxiety with stress. This implies that those with a higher level of anxiety and insomnia were not likely to have a higher level of perceived stress. The results suggest that there were possibly two types of insomniacs in this study population-one with stress-reactivity-related insomnia and another group with insomnia not related to stress. ${ }^{33}$ Most or at least a major section of students with high stress in this study might have had a stress-reactivity to sleep. This seemingly explains the observation of high-stress score also predicting insomnia in the first model. ${ }^{9,12,33}$ However, the presence of insomnia did not predict highstress scores in the second model. This is likely explained by the incidences of insomnia unrelated to stress as well. Furthermore, these data extend and support the notion that insomnia could be one pathway where psychological stress 
may confer a high risk of anxiety. The findings of the present study are thematically similar to previous studies which showed that stressful life events often precede anxiety disorders. ${ }^{34}$

There are several possible interpretations of the role that insomnia plays as a link between stress and anxiety. Firstly, poor sleep could affect an individual's ability to encode and consolidate emotional memory, ${ }^{35}$ potentially leading to poor recall capacity of declarative memories during academic life. ${ }^{36}$ Those students who are already dealing with increased perceived stress, the comorbid incidence of insomnia may further impair the memory encoding and recall during the exams, which may eventually lead to aggravated anxiety levels. Secondly, subjective poor sleep quality has been reported with increased amygdala activation, which is negatively associated with spatial and recall memory in students, ${ }^{37}$ thus, impacting their academic performance, which also possibly increases anxiety levels. Indeed, subjective poor sleep quality predicted affective dysregulation involving anxiety and depression. ${ }^{37-39}$

The present study supports the literature by suggesting the role of insomnia in the relationship between stress and anxiety development. This may be partially explained by the shared neuroendocrine mechanisms of stress reactivity and hypothalamic-pituitary and adrenal (HPA) stress axis activation. $^{40}$ Contrasting evidence shows that poor sleep ${ }^{41-44}$ and anxiety confer HPA dysregulation. ${ }^{45,46}$ Accordingly, good sleep quality may offer protection against stress and HPA axis activation. ${ }^{47}$ On the contrary, poor sleep quality makes insomniacs vulnerable to stressful events in life and increases the probability of anxiety occurrence via activation of the HPA axis. Future studies should explore the role of clinical markers of insomnia and its role in mediating stressful events and anxiety.

The etiological basis of stress is multifactorial with most of the factors involved in the expression of psychological stress being external in nature such as a tight deadline or an argument with a friend that lasts for a brief period and may subside once the situation has been resolved. ${ }^{33,48}$ On the contrary, anxiety is a reaction to a stressful situation. ${ }^{34,48}$ Intriguingly, insomnia did not mediate the effects of anxiety on stress. A plausible explanation of our findings is that students with higher perceived stress and comorbid insomnia were likely to develop anxiety, possibly through the HPA axis pathway. However, the absence of opposite relationships, ie, a higher level of anxiety and comorbid insomnia in students, was not associated with stress, possibly because of the absence of concomitant external stimuli for stress exhibition.

The role of insomnia in mediating the positive relationship between stress and anxiety has important clinical implications, one study suggested the worst clinical outcomes when stress was comorbid with anxiety in post-traumatic stress disorder (PTSD) patients. ${ }^{49}$ Moreover, estimates may vary, but studies showed that patients having a diagnosis of mental health issues like stress had shown $\sim 65 \%$ likelihood of having other comorbid mental health disorders. ${ }^{49,50}$ Consistently, each mental health disorder may exacerbate the other, impeding the patient's recovery cycle. ${ }^{51}$ Clinically, this finding supports the existing literature and optimizes the present clinical guidelines in the management of insomnia and prevent future predisposition of the patient to anxiety and other mental health disorders. Some of the commonly used behavioral interventional approaches for chronic insomnia are cognitive behavioral therapy, Mindfulness-Based Therapy in combination with behavioral techniques (MBT-I) ${ }^{52}$ Most of these treatment procedures for insomnia do help in the management of anxiety by prospective pathways, ie, by managing/ decreasing the severity of insomnia, as well as stress.

\section{Limitations}

This study presents a novel role as a mediator of insomnia in the comorbidity of stress and anxiety. This study has some limitations. Firstly, the findings of this study should be interpreted based on the cross-sectional nature of the study. A cohort study with groups of participants with stress only, stress, and co-morbid insomnia, and anxiety with co-morbid stress may help to better establish the mediator role of insomnia for elicitation of anxiety in people with psychological stress. If such a mediator role of insomnia is further validated in cohort studies, then this would highlight the need for the treatments of stress and insomnia to manage anxiety and prevent the future risks for the development of comorbid anxiety and other mental health disorders. Secondly, the relationship in this study may have been slightly influenced by the biased gender ratio. It would be interesting to investigate the mediating role of insomnia in the relation of stress and anxiety among females. This is because sex/gender-related differences have been reported in anxiety disorders and their relationship with stress. ${ }^{53}$ Additionally, not assessing 
of physical activity levels and BMI and their consequent non-consideration in the model was a limitation. ${ }^{54}$

Future studies should assess the potential differential relationship between insomnia, stress, and anxiety. However, regardless of the limitations of the present study, our findings contribute to the existing body of literature and could be potentially used to optimize clinical guidelines in the management of mental health complications in university students.

\section{Conclusion}

Students having higher perceived stress level and comorbid insomnia were also likely to have higher anxiety levels. Insomnia seemingly mediated the relationship between stress and anxiety in this crosssectional study. However, further substantiation of this mediating role of insomnia needs to be established by longitudinal studies.

\section{Data Sharing Statement}

The de-identified dataset used and/or analyzed during the current study are available from the corresponding author on reasonable request.

\section{Ethics Approval and Consent to Participate}

The Human Institutional Ethics Review Committee, College of Medicine and Health Sciences, Mizan-Tepi University, Mizan, Ethiopia approved the study. All procedures performed in studies involving human participants were in accordance with the ethical standards of the institutional and/or national research committee and with the 1964 Helsinki declaration and its later amendments or comparable ethical standards. Informed written consent was obtained from all participants prior to the commencement of the study.

\section{Consent for Publication}

Not applicable.

\section{Acknowledgments}

We are grateful to the participants of the study. The authors extend their appreciation to the Deanship of Scientific Research at Majmaah University for funding this work under Project Number No (RGP-2019-40). The authors are grateful to the Mizan-Tepi University.

\section{Funding}

The Deanship of Scientific Research at Majmaah University funded this work under Project Number No (RGP-2019-40).

\section{Disclosure}

Seithikurippu R Pandi-Perumal reports non-financial support from Somnogen Canada Inc and occasional royalties for the editorial contribution from Springer during the conduct of the study and is an employee of Somnogen Canada Inc. The authors report no other potential conflicts of interest for this work.

\section{References}

1. Bandelow B, Michaelis S. Epidemiology of anxiety disorders in the 21st century. Dialogues Clin Neurosci. 2015;17(3):327-335.

2. Fawzy M, Hamed SA. Prevalence of psychological stress, depression and anxiety among medical students in Egypt. Psychiatry Res. 2017;255:186-194. doi:10.1016/j.psychres.2017.05.027

3. Bouter Y, Brzozka MM, Rygula R, et al. Chronic psychosocial stress causes increased anxiety-like behavior and alters endocannabinoid levels in the brain of C57B1/6J Mice. Cannabis Cannabinoid Res. 2020;5(1):51-61. doi:10.1089/can.2019.0041

4. Berry A, Bellisario V, Capoccia S, et al. Social deprivation stress is a triggering factor for the emergence of anxiety- and depression-like behaviours and leads to reduced brain BDNF levels in C57BL/6J mice. Psychoneuroendocrinology. 2012;37(6):762-772. doi:10.1016/ j.psyneuen.2011.09.007

5. Almojali AI, Almalki SA, Alothman AS, Masuadi EM, Alaqeel MK. The prevalence and association of stress with sleep quality among medical students. J Epidemiol Glob Health. 2017;7(3):169-174. doi:10.1016/j.jegh.2017.04.005

6. Valerio TD, Kim MJ, Sexton-Radek K. Association of stress, general health, and alcohol use with poor sleep quality among U.S. College Students. Am J Health Educ. 2016;47(1):17-23. doi:10.1080/ 19325037.2015.1111173

7. Safhi MA, Alafif RA, Alamoudi NM, et al. The association of stress with sleep quality among medical students at King Abdulaziz University. J Fam Med Prim Care. 2020;9(3):1662-1667. doi:10.4103/jfmpc.jfmpc_745_19

8. Maskevich S, Cassanet A, Allen NB, Trinder J, Bei B. Sleep and stress in adolescents: the roles of pre-sleep arousal and coping during school and vacation. Sleep Med. 2020;66:130-138. doi:10.1016/j. sleep.2019.10.006

9. Kalmbach DA, Pillai V, Arnedt JT, Drake CL. Identifying at-risk individuals for insomnia using the Ford Insomnia Response to Stress Test. Sleep. 2016;39(2):449-456. doi:10.5665/sleep.5462

10. Drake CL, Friedman NP, Wright KP, Roth T. Sleep reactivity and insomnia: genetic and environmental influences. Sleep. 2011;34:1179-1188. doi:10.5665/SLEEP.1234

11. Fernandez-Mendoza J, Shaffer ML, Olavarrieta-Bernardino S, et al. Cognitive-emotional hyperarousal in the offspring of parents vulnerable to insomnia: a nuclear family study. $J$ Sleep Res. 2014;23:489-498. doi:10.1111/jsr.12168

12. Palagini L, Faraguna U, Mauri M, Gronchi A, Morin CM, Riemann D. Association between stress-related sleep reactivity and cognitive processes in insomnia disorder and insomnia subgroups: preliminary results. Sleep Med. 2016;19:101-107. doi:10.1016/j. sleep.2015.10.020 
13. Geoffroy PA, Hoertel N, Etain B, et al. Insomnia and hypersomnia in major depressive episode: prevalence, sociodemographic characteristics and psychiatric comorbidity in a population-based study. $J$ Affect Disord. 2018;226:132-141. doi:10.1016/j.jad.2017.09.032

14. Choueiry N, Salamoun T, Jabbour H, El Osta N, Hajj A, Rabbaa Khabbaz L. Insomnia and relationship with anxiety in university students: a cross-sectional designed study. PLoS One. 2016;11(2): e0149643. doi:10.1371/journal.pone.0149643

15. de Zambotti M, Goldstone A, Colrain IM, Baker FC. Insomnia disorder in adolescence: diagnosis, impact, and treatment. Sleep Med Rev. 2018;39:12-24. doi:10.1016/j.smrv.2017.06.009

16. Brand S, Kirov R. Sleep and its importance in adolescence and in common adolescent somatic and psychiatric conditions. Int J Gen Med. 2011;4:425-442. doi:10.2147/IJGM.S11557

17. Washington TD. Psychological stress and anxiety in middle to late childhood and early adolescence: manifestations and management. J Pediatr Nurs. 2009;24(4):302-313. doi:10.1016/j.pedn.2008.04.011

18. Wittchen HU, Kessler RC, Pfister H, Lieb M. Why do people with anxiety disorders become depressed? A prospective-longitudinal community study. Acta Psychiatr Scand Suppl. 2000;102 (406):14-23. doi:10.1111/j.0065-1591.2000.acp29-03.x

19. Kebede MA, Anbessie B, Ayano G. Prevalence and predictors of depression and anxiety among medical students in Addis Ababa, Ethiopia. Int J Ment Health Syst. 2019;13(1):30. doi:10.1186/ s13033-019-0287-6

20. Spitzer RL, Kroenke K, Williams JB, Lowe B. A brief measure for assessing generalized anxiety disorder: the GAD-7. Arch Intern Med. 2006;166(10):1092-1097. doi:10.1001/archinte.166.10.1092

21. Alghadir A, Manzar MD, Anwer S, Albougami A, Salahuddin M. Psychometric properties of the generalized anxiety disorder scale among Saudi University Male Students. Neuropsychiatr Dis Treat. 2020;16:1427-1432. doi:10.2147/NDT.S246526

22. Kroenke K, Spitzer RL, Williams JB, Monahan PO, Lowe B. Anxiety disorders in primary care: prevalence, impairment, comorbidity, and detection. Ann Intern Med. 2007;146(5):317-325.

23. Cohen S, Kamarck T, Mermelstein R. A global measure of perceived stress. J Health Soc Behav. 1983;24(4):385-396. doi:10.2307/2136404

24. Manzar MD, Salahuddin M, Peter S, et al. Psychometric properties of the perceived stress scale in Ethiopian university students. $B M C$ Public Health. 2019;19(1):41. doi:10.1186/s12889-018-6310-z

25. Tarrasch R, Laudon M, Zisapel N. Cross-cultural validation of the Leeds sleep evaluation questionnaire (LSEQ) in insomnia patients. Hum Psychopharmacol. 2003;18(8):603-610. doi:10.1002/hup.534

26. Manzar MD, Salahuddin M, Maru TT, et al. Validation of the adapted Leeds sleep evaluation questionnaire in Ethiopian university students. Health Qual Life Outcomes. 2018;16(1):49. doi:10.1186/s12955-0180876-0

27. Hameed UA, Al-Jarrah MD, Manzar MD, et al. Leeds sleep evaluation questionnaire in Jordanian university students. A psychometric investigation using comparative confirmatory factor analysis. Saudi Med J. 2020;41(7):746-752. doi:10.15537/smj.2020.7.25146

28. Baron RM, Kenny DA. The moderator-mediator variable distinction in social psychological research: conceptual, strategic, and statistical considerations. J Pers Soc Psychol. 1986;51(6):1173-1182. doi:10.1037/0022-3514.51.6.1173

29. Hayes A The PROCESS macro for SPSS and SAS (version 2.13) [Software]. 2013.

30. Bagheri Hosseinabadi M, Khanjani N, Ebrahimi MH, Haji B, Abdolahfard M. The effect of chronic exposure to extremely low-frequency electromagnetic fields on sleep quality, stress, depression and anxiety. Electromagn Biol Med. 2019;38(1):96-101. doi:10.1080/15368378.2018.1545665

31. Herbison CE, Allen K, Robinson M, Newnham J, Pennell C. The impact of life stress on adult depression and anxiety is dependent on gender and timing of exposure. Dev Psychopathol. 2017;29 (4):1443-1454. doi:10.1017/S0954579417000372
32. Lowery-Gionta EG, Crowley NA, Bukalo O, Silverstein S, Holmes A, Kash TL. Chronic stress dysregulates amygdalar output to the prefrontal cortex. Neuropharmacology. 2018;139:68-75. doi:10.1016/j.neuropharm.2018.06.032

33. Drake CL, Pillai V, Roth T. Stress and sleep reactivity: a prospective investigation of the stress-diathesis model of insomnia. Sleep. 2014;37(8):1295-1304. doi:10.5665/sleep.3916

34. Faravelli C, Pallanti S. Recent life events and panic disorder. Am J Psychiatry. 1989;146(5):622-626.

35. Hu P, Stylos-Allan M, Walker MP. Sleep facilitates consolidation of emotional declarative memory. Psychol Sci. 2006;17(10):891-898. doi:10.1111/j.1467-9280.2006.01799.x

36. Van Vreeswijk MF, De Wilde EJ. Autobiographical memory specificity, psychopathology, depressed mood and the use of the Autobiographical Memory Test: a meta-analysis. Behav Res Ther. 2004;42(6):731-743. doi:10.1016/S0005-7967(03)00194-3

37. Prather AA, Bogdan R, Hariri AR. Impact of sleep quality on amygdala reactivity, negative affect, and perceived stress. Psychosom Med. 2013;75(4):350-358. doi:10.1097/PSY.0b013e31828ef15b

38. Franzen PL, Buysse DJ, Rabinovitz M, Pollock BG, Lotrich FE. Poor sleep quality predicts onset of either major depression or subsyndromal depression with irritability during interferon-alpha treatment. Psychiatry Res. 2010;177(1-2):240-245. doi:10.1016/j. psychres.2009.02.011

39. Mauss IB, Troy AS, LeBourgeois MK. Poorer sleep quality is associated with lower emotion-regulation ability in a laboratory paradigm. Cogn Emot. 2013;27(3):567-576. doi:10.1080/ 02699931.2012 .727783

40. Chrousos GP. Stress and disorders of the stress system. Nat Rev Endocrinol. 2009;5(7):374-381.

41. Adam K, Tomeny M, Oswald I. Physiological and psychological differences between good and poor sleepers. J Psychiatr Res. 1986;20(4):301-316. doi:10.1016/0022-3956(86)90033-6

42. Vgontzas AN, Tsigos C, Bixler EO, et al. Chronic insomnia and activity of the stress system: a preliminary study. J Psychosom Res. 1998;45(1):21-31. doi:10.1016/S0022-3999(97)00302-4

43. Rodenbeck A, Huether G, Ruther E, Hajak G. Interactions between evening and nocturnal cortisol secretion and sleep parameters in patients with severe chronic primary insomnia. Neurosci Lett. 2002;324(2):159-163. doi:10.1016/S0304-3940(02)00192-1

44. Riemann D, Klein T, Rodenbeck A, et al. Nocturnal cortisol and melatonin secretion in primary insomnia. Psychiatry Res. 2002;113 (1-2):17-27. doi:10.1016/S0165-1781(02)00249-4

45. Juruena MF, Eror F, Cleare AJ, Young AH. The role of early life stress in HPA axis and anxiety. Adv Exp Med Biol. 2020;1191:141-153.

46. Graeff FG, Garcia-Leal C, Del-Ben CM, Guimaraes FS. Does the panic attack activate the hypothalamic-pituitary-adrenal axis? An Acad Bras Cienc. 2005;77(3):477-491. doi:10.1590/S000137652005000300009

47. Buckley TM, Schatzberg AF. On the interactions of the hypothalamic-pituitary-adrenal (HPA) axis and sleep: normal HPA axis activity and circadian rhythm, exemplary sleep disorders. J Clin Endocrinol Metab. 2005;90(5):3106-3114. doi:10.1210/jc.2004-1056

48. Schneiderman N, Ironson G, Siegel SD. Stress and health: psychological, behavioral, and biological determinants. Annu Rev Clin Psychol. 2005;1:607-628. doi:10.1146/annurev. clinpsy.1.102803.144141

49. Hatch R, Young D, Barber V, Griffiths J, Harrison DA, Anxiety WP. Depression and post traumatic stress disorder after critical illness: a UK-wide prospective cohort study. Crit Care. 2018;22(1):310. doi:10.1186/s13054-018-2223-6

50. Hammond NG, Orr SL, Colman I. Early life stress in adolescent migraine and the mediational influence of symptoms of depression and anxiety in a Canadian Cohort. Headache. 2019;59 (10):1687-1699. doi:10.1111/head.13644 
51. Staner L. Comorbidity of insomnia and depression. Sleep Med Rev. 2010;14(1):35-46. doi:10.1016/j.smrv.2009.09.003

52. Ree M, Junge M, Cunnington D. Australasian Sleep Association position statement regarding the use of psychological/behavioral treatments in the management of insomnia in adults. Sleep Med. 2017;36(Suppl 1):S43-S47. doi:10.1016/j.sleep.2017.03.017
53. Maeng LY, Milad MR. Sex differences in anxiety disorders: interactions between fear, stress, and gonadal hormones. Horm Behav. 2015;76:106-117. doi:10.1016/j.yhbeh.2015.04.002

54. Hargens TA, Kaleth AS, Edwards ES, Butner KL. Association between sleep disorders, obesity, and exercise: a review. Nat Sci Sleep. 2013;5:27. doi:10.2147/NSS.S34838

\section{Publish your work in this journal}

Nature and Science of Sleep is an international, peer-reviewed, open access journal covering all aspects of sleep science and sleep medicine, including the neurophysiology and functions of sleep, the genetics of sleep, sleep and society, biological rhythms, dreaming, sleep disorders and therapy, and strategies to optimize healthy sleep.
The manuscript management system is completely online and includes a very quick and fair peer-review system, which is all easy to use. Visit http://www.dovepress.com/testimonials.php to read real quotes from published authors. 